\title{
Hyaluronan as a Promising Biomarker for Myocardial Damage
}

\author{
Goktug Savas, ${ }^{1}$ Nihat Kalay, ${ }^{2}$ Pinar Altin, ${ }^{3}$ Gamze Karadas Dursun, ${ }^{3}$ Murat Cetin ${ }^{2}$ \\ and Metin Aytekin ${ }^{4}$ \\ ${ }^{1}$ Department of Cardiology, Siyami Ersek Thoracic and Cardiovascular Surgery Center Training and Research \\ Hospital, Istanbul, Turkey \\ ${ }^{2}$ Department of Cardiology, Erciyes University School of Medicine, Kayseri, Turkey \\ ${ }^{3}$ Department of Medical Biology, Erciyes University School of Medicine, Kayseri, Turkey \\ ${ }^{4}$ Department of Pathobiology, Lerner Research Institute, Cleveland Clinic, Cleveland, OH, USA
}

Hyaluronan is a glycosaminoglycan, one of the chief components of the extracellular matrix. The aim of the present study is to investigate plasma hyaluronan levels among patients with acute myocardial infarction (AMI). This prospective study enrolled 56 consecutive patients with AMI who underwent percutaneous coronary intervention within 2 hours after admission. Plasma levels of hyaluronan were measured at the time of admission (baseline), and on the 7th and 30th day after AMI. Echocardiographic examinations were performed at baseline and on the 30th day after AMI. The hyaluronan levels were $33.2 \pm 3.1 \mathrm{ng} / \mathrm{ml}$ (mean $\pm \mathrm{SD})$ at baseline, increased on the 7th day $(46.2 \pm 5.9 \mathrm{ng} / \mathrm{ml})$, and continued to remain high on the 30th day after AMI $(50.1 \pm 5.1 \mathrm{ng} / \mathrm{ml})$. There were significant correlations of the hyaluronan levels between baseline and on the 7th day $(r=0.535, p<0.001)$ and between baseline and on the 30th day $(r=0.263, p$ $=0.05$ ). Significant correlations were also found between hyaluronan levels on the 30th day, and the peak levels of CK-MB $(r=0.429, p=0.001)$ or highly sensitive troponin levels $(r=0.360, p=0.006)$. The hyaluronan levels were significantly higher on the 30th day after AMI in patients with anterior infarction, but not in patients with non-anterior infarction ( $p=0.01$ vs. $p=0.653$ ). In conclusion, this is the first report that demonstrates the increase of plasma hyaluronan levels among patients with AMI.

Keywords: acute myocardial infarction; biomarker; extra cellular matrix; hyaluronan; myocardial damage Tohoku J. Exp. Med., 2019 June, 248 (2), 99-106. C 2019 Tohoku University Medical Press

\section{Introduction}

Following acute myocardial infarction (AMI), a cascade of compensatory structural events occurs within the myocardium and contributes to eventual left ventricular (LV) dysfunction. These biological changes in the diseased heart, also termed myocardial remodeling, are a summation of both cellular (myocyte growth, apoptosis, and necrosis) and extracellular processes (Anversa et al. 1991; Chareonthaitawee et al. 1995; Spinale 2007).

The extracellular processes, which include dynamic changes in the extracellular matrix (ECM), are characterized by remodeling of the connective tissue and synthesis of collagen (Burlew and Weber 2000; Spinale 2007). During the process, myofibroblasts accumulate in the injured myocardium, deposit ECM proteins, and form a collagen-based scar (Frangogiannis 2006). The collagen deposition helps to maintain the mechanical strength of the affected ventricular wall. It is important to have a balance of ECM production and turnover, which is fundamental in post-infarction healing of the cardiac wound (Zimmermann
2015).

Hyaluronan (HA), a glycosaminoglycan, is critical to the assembly and structure of the ECM. HA provides rigidity and serves a scaffolding function for the matrix (Burlew and Weber 2000; Triggs-Raine and Natowicz 2015). The hydrophilic groups of HA attract water to hydrate tissues and this facilitates cellular movement and proliferation, which are critical for cellular migrations during the remodeling of the tissues (Toole et al. 2002; Triggs-Raine and Natowicz 2015). The role of HA in cardiac wound healing has been investigated. In a rat model of myocardial infarction, Waldenstrom et al. (1991) demonstrated that the HA content of infarcted myocardium increased progressively and reached a threefold increase on the third day after the ligation of left anterior descending artery, compared with non-infarcted myocardium in sham-operated rats. By contrast, the role of HA has been rarely studied in patients with heart disease. Increased plasma levels of HA were shown in patients with mitral stenosis or pulmonary hypertension (Aytekin et al. 2008; Kalay et al. 2014). However, to the best of our knowledge, there is no published study that has

Received November 26, 2018; revised and accepted May 31, 2019. Published online June 25, 2019; doi: 10.1620/tjem.248.99.

Correspondence: Goktug Savas, M.D., Department of Cardiology, Siyami Ersek Thoracic and Cardiovascular Surgery Center Training and Research Hospital, No. 13 Tibbiye Street, Uskudar, Istanbul 34668, Turkey.

e-mail: goktug_savas@hotmail.com 
investigated HA levels in patients with AMI. The present study aims to investigate plasma HA levels and its kinetics in patients with AMI.

\section{Material and Methods}

Study population

Fifty-six AMI patients who underwent percutaneous coronary intervention within 2 hours after admission were prospectively enrolled in this study between October 2012 and July 2014. Erciyes University ethics committee approved the study, and informed consent was obtained from all the patients. Exclusion criteria for the study were as follows: chronic obstructive pulmonary disease, any renal disorder (serum creatinine $>1.3 \mathrm{mg} / \mathrm{dl}$ ), a history of pulmonary thromboembolism or pulmonary hypertension, heart failure, morbid obesity, myopericarditis, and muscular injection or trauma within the last 24 hours. AMI was defined by a positive troponin test and elevated cardiac markers with typical chest pain or electrocardiographic changes consistent with non-ST elevation (NSTEMI) or ST elevation myocardial infarction (STEMI) (Thygesen et al. 2012).

The patients were defined as anterior infarction if they had had a persistent ST-segment elevation $\geq 0.1 \mathrm{mV}$ in at least two of six precordial leads or a new left bundle-branch block. Patients who had a persistent ST-segment elevation $\geq 0.1 \mathrm{mV}$ in at least two of leads II, III, and aVF were defined as inferior infarction while patients with a persistent ST-segment elevation of at least $0.1 \mathrm{mV}$ in leads I and aVL were defined as high lateral infarction (Zimetbaum and Josephson 2003).

\section{Echocardiography}

Each patient underwent transthoracic echocardiography (TTE) with a $2.5 \mathrm{MHz}$ transducer for TTE (Vivid $7^{\circledR}$ GE Medical System, Horten, Norway) on the first day. On the 30th day after AMI, each patient was re-evaluated with TTE to determine any changes. All patients underwent the procedure in the left lateral recumbent position after a 10-min resting period. The Simpson's method in the apical four-chamber view was used to assess LVEF as recommended by the guidelines (Nagueh et al. 2009). From the parasternal long axis, via M-mode, LV end-diastolic diameter (LVEDD), LV end-systolic diameter (LVESD), left atrium diameter, interventricular septum thicknesses, and posterior wall thicknesses were recorded.

\section{Biochemical and hematological measurements}

Antecubital venous blood samples were drawn from all the patients upon the time of admission before the percutaneous coronary intervention and seven days after the percutaneous coronary intervention. The blood samples for the measurement of creatine phosphokinase $\mathrm{MB}$ (CKMB) and highly sensitive troponin-I were serially obtained at the baseline, and the 6th and 24th hours. The levels of glucose, blood urea nitrogen, creatinine, lipid profile, liver function tests, and uric acid were determined by Thermo Clinical Lab Systems (Thermo Clinical Lab systems, Vantaa, Finland). Hemoglobin, hematocrit, platelet and white blood cell (WBC) count were determined using a blood counter (Sysmex K-1000, Sysmex Medica Co., Kobe, Japan). Highly sensitive troponin-I was measured with the ADVIA Centaur Troponin I-Ultra assay (Siemens Healthcare Diagnostics Inc., Munich, Germany). The analytic sensitivity of the assay is $0.006 \mathrm{pg} /$ $\mathrm{ml}$ (or $6 \times 10^{-6} \mathrm{ng} / \mathrm{ml}$ ). On the 30th day after AMI, blood samples were again drawn from the antecubital vein. The blood samples were kept at $-80^{\circ} \mathrm{C}$ until further analysis.

\section{Measurement of HA}

The levels of HA in the plasma were determined by an enzymelinked binding protein assay for the determination of HA (Corgenix Inc., Broomfield, CO, USA) (Haserodt et al. 2011). The system uses a capture molecule known as HA binding protein. The optical densities were measured at $450 \mathrm{~nm}$. All the test procedures were carried out using the manufacturer's instructions.

\section{Statistical analysis}

The SPSS statistical package for Windows, version 15.0 (SPSS Inc., Chicago, IL, USA) was used for statistical analysis. The data were presented as a mean \pm standard deviation (SD). The categorical variables were presented as numbers and percentages. The Kolmogorov-Smirnov test was used to evaluate if the distribution of variables was normal. Continuous variables between groups were compared with a t-test or Mann Whitney U, while categorical variables were compared with a chi-square test. Pearson or Spearman tests were used to analyze the correlation between variables. A p value of less than 0.05 was considered statistically significant. All reported $\mathrm{p}$ values were two-sided.

\section{Results}

\section{Characteristics of the subjects}

Fifty-six patients who underwent a percutaneous coronary intervention with the diagnosis of AMI were enrolled in the present study. The demographic characteristics of patients are shown in Table 1. The mean age of participants was $55.6 \pm 9.4$ years and most were male (85\%). Fortynine patients $(87.5 \%)$ had an acute coronary syndrome with ST-segment elevation and seven patients (12.5\%) had an acute coronary syndrome without ST-segment elevation. The right coronary artery (RCA) was the most frequently detected culprit lesion (29 patients, 51.8\%). Twenty-one patients had left anterior descending (LAD) culprit lesions (37.5\%), and the remaining six patients $(10.7 \%)$ had a circumflex artery (CX) lesion. Forty patients (71.4\%) were treated with drug-eluting stents and the rest of the 16 patients were treated with bare-metal stents. At the time of discharge from hospital, all of the recruited patients were on dual anti-platelet and statin therapy, 55 patients were on a beta blocker, and 53 of them were on an ACE inhibitor. Diuretics were prescribed to 3 patients.

The echocardiography results of the recruited patients at baseline and on the 30th day after AMI are presented in Table 2. The left ventricular ejection fraction was $53.1 \pm$ $7.6 \%$ at baseline, and was $55.2 \pm 7.2 \%$ on the 30 th day after AMI $(p=0.02)$. The left ventricular systolic and diastolic diameters were not significantly changed on the 30th day after AMI ( $p=0.92$ and $p=0.63$, respectively).

\section{Plasma HA levels}

Plasma levels of HA in the recruited patients are shown in Fig. 1. Plasma HA levels were $33.2 \pm 3.1 \mathrm{ng} / \mathrm{ml}$ (mean $\pm \mathrm{SD}$ ) at baseline, and significantly increased to 46.2 $\pm 5.9 \mathrm{ng} / \mathrm{ml}$ on the 7 th day after AMI $(\mathrm{p}=0.014)$. The HA levels continued to remain high $(50.1 \pm 5.1 \mathrm{ng} / \mathrm{ml})$ on the 30th day after AMI, and were significantly higher compared 
Table 1. The demographic characteristics of 56 patients with acute myocardial infarction.

\begin{tabular}{|c|c|}
\hline Variables & \\
\hline Age (years) & $55.6 \pm 9.4$ \\
\hline Male n $(\%)$ & $48(85)$ \\
\hline Body mass index (kg/m2) & $29.5 \pm 3.8$ \\
\hline Blood Urea Nitrogen (mg/dl) & $15.9 \pm 4.9$ \\
\hline Creatine (mg/dl) & $0.92 \pm 0.2$ \\
\hline \multicolumn{2}{|l|}{ Cholesterol (mg/dl) } \\
\hline Total & $182 \pm 29$ \\
\hline HDL & $34.6 \pm 6.3$ \\
\hline LDL & $115.9 \pm 28.8$ \\
\hline Triglyceride (mg/dl) & $159.3 \pm 107.8$ \\
\hline White blood cell $(\mathrm{WBC})\left(\times 10^{9} / \mathrm{L}\right)$ & $11.9 \pm 3.5$ \\
\hline Hemoglobin (g/dL) & $14.5 \pm 2.1$ \\
\hline Mean corpuscular volume (MCV) & $88.7 \pm 6.3$ \\
\hline Coronary artery disease $\mathrm{n}(\%)$ & $9(16)$ \\
\hline Hypertension $\mathrm{n}(\%)$ & $26(46)$ \\
\hline Diabetes Mellitus n (\%) & $9(16)$ \\
\hline Smoking n (\%) & $45(80.4)$ \\
\hline \multicolumn{2}{|l|}{ Diagnosis n (\%) } \\
\hline NSTEMI n (\%) & $7(12.5)$ \\
\hline STEMI n (\%) & $49(87.5)$ \\
\hline -ST segment elevation in anterior leads or LBBB & $19(33.9)$ \\
\hline -ST segment elevation in inferior leads & $27(48.2)$ \\
\hline -ST segment elevation in high lateral or posterior leads & $3(5.35)$ \\
\hline
\end{tabular}

Data are expressed as mean \pm SD or numbers (percentages) of patients [n (\%)].

NSTEMI, non-ST segment myocardial infarction; STEMI, ST segment myocardial infarction; LBBB, left bundle branch block.

to baseline $(p=0.002)$ (Fig. 1). However, there was no significant difference in HA levels between the 7th and 30th days $(p=0.589)$. There were significant correlations of the HA levels between baseline and on the 7th day $(r=0.535, p$ $<0.001)$ and between baseline and on the 30th day $(\mathrm{r}=$ $0.263, \mathrm{p}=0.05$ ).

Plasma levels of HA were increased more than $50 \%$ in
20 patients (Table 3 ). When the patients with HA elevation $<50 \%$ were compared with the patients who had elevated HA levels more than $50 \%$, those with elevated HA levels $(50 \%<)$ had higher numbers of neutrophils $(9.10 \pm 3.16 \mathrm{vs}$. $6.70 \pm 2.68, \mathrm{p}=0.009$ ), lower numbers of lymphocytes $(1.79 \pm 0.77$ vs. $2.64 \pm 1.56, p=0.026)$, higher neutrophil/ lymphocyte ratio (NLR) $(6.46 \pm 5.24$ vs. $3.66 \pm 2.46, \mathrm{p}=$ 
Table 2. Echocardiographic parameters of 56 patients at baseline and on the 30th day after AMI.

\begin{tabular}{|l|l|l|l|}
\hline & Baseline & 30th day after AMI & p value \\
\hline LVSD $(\mathrm{mm})$ & $33.2 \pm 5.1$ & $33.1 \pm 4.9$ & 0.92 \\
\hline LVDD $(\mathrm{mm})$ & $48.4 \pm 4.8$ & $48.4 \pm 5.0$ & 0.63 \\
\hline LA diameter $(\mathrm{mm})$ & $34.4 \pm 3.7$ & $34.9 \pm 3.9$ & 0.18 \\
\hline LVEF (\%) & $53.1 \pm 7.6$ & $55.2 \pm 7.2$ & 0.02 \\
\hline
\end{tabular}

Data are expressed as mean \pm SD.

LVSD, left ventricle systolic diameter; LVDD, left ventricle diastolic diameter; LA, left atrium; LVEF, left ventricle ejection fraction.

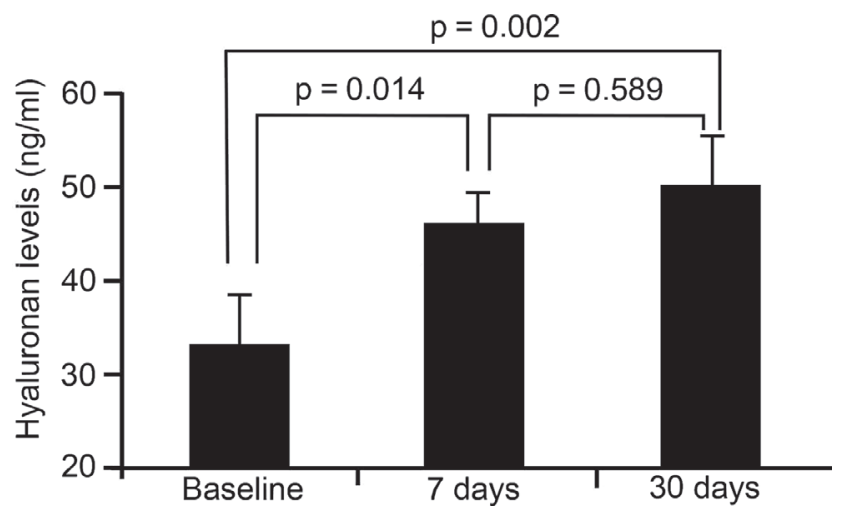

Fig. 1. Time-related changes in plasma hyaluronan levels in patients with acute myocardial infarction.

The data represent plasma hyaluronan levels in 56 patients with acute myocardial infarction (AMI) at baseline and the levels on 7 days (7th day) and 30 days (30th day) after AMI (mean $\pm \mathrm{SD}$ ). The hyaluronan level was increased from $33.2 \pm 3.1 \mathrm{ng} / \mathrm{ml}$ at baseline to $46.2 \pm 5.9$ $\mathrm{ng} / \mathrm{ml}$ on the 7 th day, and continued to remain high on the 30 th day after AMI $(50.1 \pm 5.1 \mathrm{ng} / \mathrm{ml})$.

$0.011)$, and higher age $(57.7 \pm 9.2$ vs. $52.7 \pm 9.1, \mathrm{p}=0.005)$ (Table 3).

There were also significant correlations between the plasma HA levels on the 30th day after AMI and the peak levels of CK (Fig. 2A, r $=0.377, p=0.004$ ), CKMB (Fig. $2 \mathrm{~B}, \mathrm{r}=0.429, \mathrm{p}=0.001$ ), highly sensitive troponin-I (Fig. $2 \mathrm{C}, \mathrm{r}=0.360, \mathrm{p}=0.006$ ), aspartate aminotransferase (AST) (Fig. $2 \mathrm{D}, \mathrm{r}=0.429, \mathrm{p}=0.001$ ), or lactate dehydrogenase $(\mathrm{LDH})($ data not shown, $\mathrm{r}=0389, \mathrm{p}=0.005)$.

\section{Relationship between echocardiographic findings and plasma HA levels}

When the patients with inferior or high lateral infarction were compared with the patients who had an anterior infarction, the latter had a lower LVEF at the baseline and on the 30th day after AMI (baseline $57.1 \pm 5.3 \%$, on the 30th day after AMI $48.2 \pm 10.1 \%, \mathrm{p}=0.001)$. In addition, HA levels were significantly higher on the 30th day after AMI $(57.9 \pm 3.7 \mathrm{ng} / \mathrm{ml})$ than baseline $(43.9 \pm 9.0 \mathrm{ng} / \mathrm{ml})$ in patients with anterior infarction, whereas such an elevation was not found in patients with non-anterior infarction (baseline levels of $46.1 \pm 7.6 \mathrm{ng} / \mathrm{ml}$ vs. $40.5 \pm 3.5 \mathrm{ng} / \mathrm{ml}$ on the 30th day after AMI, $\mathrm{p}=0.653$ ) (Fig. 3).

\section{Discussion}

In the present study, we investigated plasma levels of HA and its kinetics in patients with AMI, and showed that plasma HA levels were significantly higher in patients with AMI compared with healthy subjects, whose plasma HA levels were reported in our previous reports (Aytekin et al. 2008; Kalay et al. 2014). Plasma HA levels increased progressively after the onset of AMI and continued to remain high on the 30th day after AMI. There were also significant correlations between the HA levels on the 30th day and the peak levels of CK, CKMB, AST, LDH, and highly sensitive troponin-I. Overall, the present findings are consistent with existing knowledge on extreme HA synthesis in the myocardium in rats with myocardial infarction (Waldenstrom et al. 1991).

In AMI, the ischemic field is subject to an inflammatory response with the emergence of leukocytes and the formation of edema. This environment is the initial phase for subsequent repair, which is characterized by the remodeling of the connective tissue and synthesis of collagen (Frangogiannis 2006). Available data indicate that HA, a high molecular-weight glycosaminoglycan and one chief component of the extracellular matrix, accumulates in affected tissues in the early stage of myocardial ischemia, and forms reversible linkages with water giving a specific contribution to lubrication and strength (Waldenstrom et al. 1991; Toole et al. 2002; Triggs-Raine and Natowicz 2015). Indeed, not only the heart but also some various organs show the similar response of an excessive accumulation of HA with injury (Bjermer et al. 1987, Nettelbladt et al. 1989). Owing to its relevant biological properties, there has been increasing interest in HA molecules in the preclinical setting. Hyaluronidase, an enzyme that catalyzes the degradation of HA, was reported to be beneficial in limiting cellular damage during myocardial ischemia in rats. It was speculated that hyaluronidase reduces tissue water accumulation, thus reducing tissue pressure and allowing 
Table 3 Clinical and biochemical variables in 20 patients with elevation $>50 \%$ in plasma hyaluronan levels (HA elevation $>50 \%$ ), and 36 patients with $\mathrm{HA}$ elevation $<50 \%$.

\begin{tabular}{|c|c|c|c|}
\hline & $\begin{array}{l}\text { HA elevation }>50 \% \\
\qquad(\mathrm{n}=20)\end{array}$ & $\begin{array}{c}\text { HA elevation }<50 \% \\
(\mathrm{n}=36)\end{array}$ & $\mathrm{p}$ value \\
\hline Age (years) & $57.7 \pm 9.2$ & $52.7 \pm 9.1$ & $0.005^{*}$ \\
\hline Male $(\%)$ & $80 \%(16)$ & $81 \%(29)$ & 0.718 \\
\hline Hypertension & $35 \%(7)$ & $47 \%(17)$ & 0.444 \\
\hline Diabetes mellitus & $20 \%(4)$ & $14 \%(5)$ & 0.495 \\
\hline Uric acid $(\mathrm{mg} / \mathrm{dL})$ & $5.40 \pm 1.52$ & $5.46 \pm 1.51$ & 0.884 \\
\hline White blood cell $\left(\times 10^{9} / \mathrm{L}\right)$ & $11.76 \pm 3.31$ & $10.52 \pm 2.60$ & 0.125 \\
\hline Hemoglobin $(\mathrm{g} / \mathrm{dL})$ & $14.53 \pm 2.25$ & $14.56 \pm 2.12$ & 0.961 \\
\hline Platelet $\left(\times 10^{9} / \mathrm{L}\right)$ & $277 \pm 97$ & $253 \pm 71$ & 0.136 \\
\hline Neutrophil $\left(\times 10^{9} / \mathrm{L}\right)$ & $9.10 \pm 3.16$ & $6.70 \pm 2.68$ & $0.009^{*}$ \\
\hline Lymphocyte $\left(\times 10^{9} / \mathrm{L}\right)$ & $1.79 \pm 0.77$ & $2.64 \pm 1.56$ & $0.026^{*}$ \\
\hline NLR & $6.46 \pm 5.24$ & $3.66 \pm 2.46$ & $0.011^{*}$ \\
\hline $\mathrm{CRP}(\mathrm{mg} / \mathrm{dL})$ & $32.05 \pm 4.76$ & $28.27 \pm 6.25$ & 0.808 \\
\hline Troponin (ng/mL) & $17.66 \pm 3.25$ & $17.68 \pm 10.02$ & 0.997 \\
\hline Troponin, peak levels $(\mathrm{ng} / \mathrm{mL})$ & $35 \pm 19.9$ & $36.32 \pm 19.3$ & 0.815 \\
\hline CPK (U/L) & $1179 \pm 565$ & $847 \pm 503$ & 0.303 \\
\hline CKMB (IU/L) & $123 \pm 69$ & $107 \pm 66$ & 0.628 \\
\hline
\end{tabular}

Data are expressed as mean \pm SD or numbers (percentages) of patients [n (\%)]. $*$ p values $<0.05$.

NLR, neutrophil/lymphocyte ratio; CRP, C-reactive protein; CPK, creatine phosphokinase; CKMB, creatine phosphokinase MB.

better perfusion (Rovetto 1977).

Kolodgie et al. (2002) examined culprit plaques from sudden coronary death patients, documented intense immunostaining positivity for $\mathrm{HA}$ and its receptor CD44 in eroded plaque and within the overlying thrombus. Recently, Pedicino et al. (2018) investigated the role of HA, CD44, and hyaluronidase-2 (HYAL2) (an enzyme that degrading high-molecular-weight HA to its pro-inflammatory $20-\mathrm{kDa}$ isoform) in the pathogenesis of acute coronary syndromes and revealed altered HA metabolism in acute coronary syndromes patients. Furthermore, they found that HYAL2 gene expression level was higher when compared with that of observed in stable angina patients. Thus, the investigators concluded that HYAL2 and HA receptors played an important role in acute coronary syndromes, in particular when associated with plaque erosion (Pedicino et al. 2018).

Overall, the broad spectrum of HA-mediated functions in cardiac wound healing raised questions. What about the plasma levels of HA during myocardial infarction and during regeneration after the infarction? Could HA be a marker of myocardial damage? The present study appears to be the first clinical study to answer these questions.

Since the role of inflammation has been recognized in cardiovascular disease, increasing attention has been paid towards investigating the relationship between NLR and coronary heart disease (Ross 1999; Nunez et al. 2008; Bolca et al. 2015; Kurtul et al. 2015; Wada et al. 2017). Several studies have shown the association of NLR with adverse outcomes in patients with coronary artery disease (Bolca et al. 2015; Kurtul et al. 2015). NLR was found to be a predictor of cardiac mortality in patients with stable 

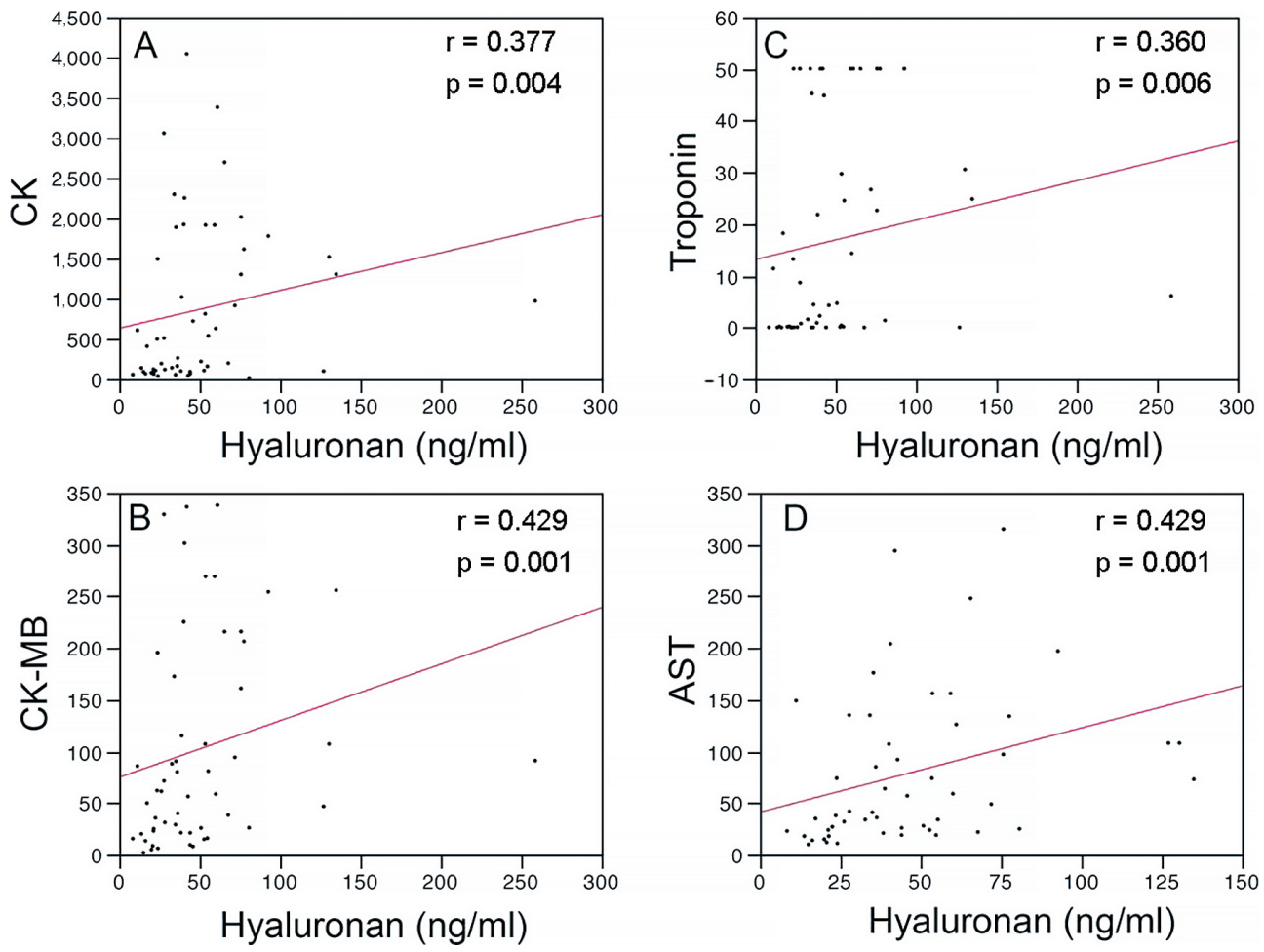

Fig. 2. The correlations of plasma hyaluronan levels with the levels of CK, CKMB, highly sensitive troponin-I, and aspartate aminotransferase.

Each panel shows the correlation of plasma hyaluronan levels with the peak levels of CK in 56 patients (A) $(r=0.377, p$ $=0.004)$, CKMB in 56 patients $(B)(r=0.429, p=0.001)$, highly sensitive troponin-I in 56 patients $(C)(r=0.360, p=$ $0.006)$, or aspartate aminotransferase (AST) in 52 patients (D) $(r=0.429, p=0.001)$ on the 30th day after acute myocardial infarction.

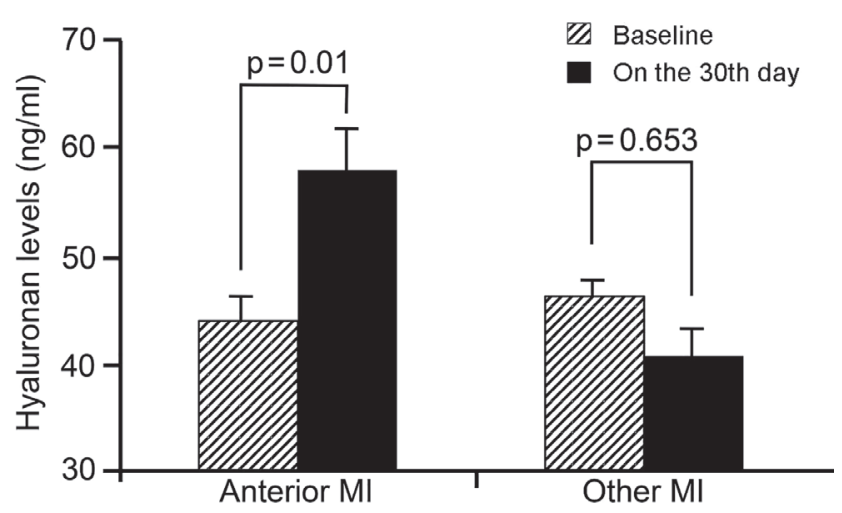

Fig. 3. Plasma hyaluronan levels in 29 patients with anterior myocardial infarction and 27 patients with inferior or high lateral myocardial infarction.

The hyaluronan levels at baseline and on the 30th day after acute myocardial infarction (AMI) are shown. The hyaluronan levels were significantly higher on the 30th days after AMI than baseline in patients with anterior infarction (Anterior MI), but not in patients with inferior or high lateral myocardial infarction (Other MI) $(\mathrm{p}=0.01$ vs. $\mathrm{p}=0.653$ ).

coronary artery disease and with acute coronary syndromes (Nunez et al. 2008; Wada et al. 2017). Interestingly, in the present study, when the patients with HA elevation $<50 \%$ group were compared with the patients who had an elevated
HA level more than $50 \%$, the latter had a higher baseline WBC count and NLR. It could be speculated that increased plasma levels of HA may reflect the severity of the disease and the adverse outcomes in patients with AMI. In this regard, further studies are needed to weigh the prognostic value of plasma HA levels among acute coronary syndrome patients.

One of the remarkable findings of the present study is that patients experiencing an anterior wall infarction had a markedly higher plasma level of HA than patients with inferior or lateral wall infarction. It is plausible that patients with anterior myocardial infarction had a significantly larger myocardium at risk of infarction. In the present study, plasma HA levels are associated with the peak levels of CK, CKMB and troponin, all of which are the accepted biomarkers for diagnosing myocardial injury (Muller et al. 1985; Antman et al. 1996). It was also plausible that large infarcts of the left ventricle had higher absolute levels of enzyme release for proportionally restricted infarct sizes. These correlations have raised an important hypothesis: plasma HA levels might be associated with the extent of cardiomyocyte injury following AMI. Further studies with a larger number of patients by means of singlephoton emission computed tomography or magnetic resonance imaging could provide more definitive evidence.

The correlation of plasma HA levels with accepted 
markers of cardiomyocyte injury suggested that HA had a consistent role in the pathology of AMI. Thus, assessment of plasma HA levels may provide a novel valuable tool to clarify underlying pathophysiology, the extent of cardiomyocyte injury, and remodeling of the cardiac tissue following AMI. A strategy combining HA and usual biomarkers may contribute to additional information about the regeneration of ischemic myocardium. In this regard, further studies are required.

The main limitation of our study was the limited number of enrolled patients and the study conclusions need confirmation with larger studies. Moreover, we did not evaluate the association between the plasma HA levels and major adverse events. Measure of the performance of the plasma HA levels on predicting outcomes like discrimination, calibration, and reclassification were not addressed in the present study.

In conclusion, this is the first report which demonstrates that plasma HA levels increase in patients with AMI. In addition, the patients experiencing an anterior wall infarction had a markedly higher plasma level of HA than the patients with inferior or lateral wall infarction. Our results suggest that HA is a promising marker of myocardial damage. We believe that plasma HA might be a promising target to predict cardiovascular function after an acute coronary syndrome.

\section{Acknowledgments}

This work was supported in part by Erciyes University Scientific Research Fund EU/BAP, Grant number: TSA-12-3985, and by a grant from Pharmactive Pharmaceutical Company.

\section{Conflict of Interest}

The authors declare no conflict of interest.

\section{References}

Antman, E.M., Tanasijevic, M.J., Thompson, B., Schactman, M., McCabe, C.H., Cannon, C.P., Fischer, G.A., Fung, A.Y., Thompson, C., Wybenga, D. \& Braunwald, E. (1996) Cardiac-specific troponin I levels to predict the risk of mortality in patients with acute coronary syndromes. N. Engl. J. Med., 335, 1342-1349.

Anversa, P., Olivetti, G. \& Capasso, J.M. (1991) Cellular basis of ventricular remodeling after myocardial infarction. Am. J. Cardiol., 68, 7D-16D.

Aytekin, M., Comhair, S.A., de la Motte, C., Bandyopadhyay, S.K., Farver, C.F., Hascall, V.C., Erzurum, S.C. \& Dweik, R.A. (2008) High levels of hyaluronan in idiopathic pulmonary arterial hypertension. Am. J. Physiol. Lung Cell Mol. Physiol., 295, L789-799.

Bjermer, L., Engstrom-Laurent, A., Lundgren, R., Rosenhall, L. \& Hallgren, R. (1987) Hyaluronate and type III procollagen peptide concentrations in bronchoalveolar lavage fluid as markers of disease activity in farmer's lung. Br. Med. J. (Clin. Res. Ed.), 295, 803-806.

Bolca, O., Gungor, B., Ozcan, K.S., Karadeniz, F.O., Sungur, A., Koroglu, B., Bakhshyaliyev, N., Yelgec, N.S., Karatas, B., Ipek, G., Yilmaz, H. \& Ozturk, R. (2015) The neutrophil-tolymphocyte ratio is associated with bare-metal stent restenosis in STEMI patients treated with primary PCI. Coron. Artery
Dis., 26, 402-408.

Burlew, B.S. \& Weber, K.T. (2000) Connective tissue and the heart. Functional significance and regulatory mechanisms. Cardiol. Clin., 18, 435-442.

Chareonthaitawee, P., Christian, T.F., Hirose, K., Gibbons, R.J. \& Rumberger, J.A. (1995) Relation of initial infarct size to extent of left ventricular remodeling in the year after acute myocardial infarction. J. Am. Coll. Cardiol., 25, 567-573.

Frangogiannis, N.G. (2006) The mechanistic basis of infarct healing. Antioxid. Redox Signal., 8, 1907-1939.

Haserodt, S., Aytekin, M. \& Dweik, R.A. (2011) A comparison of the sensitivity, specificity, and molecular weight accuracy of three different commercially available Hyaluronan ELISA-like assays. Glycobiology, 21, 175-183.

Kalay, N., Elcik, D., Savas, G., Altin, P., Sakalar, C., Kaya, O. \& Aytekin, M. (2014) Elevated hyaluronan levels in patients with rheumatic mitral stenosis and pulmonary arterial thromboembolism. Heart Lung Circ., 23, 649-654.

Kolodgie, F.D., Burke, A.P., Farb, A., Weber, D.K., Kutys, R., Wight, T.N. \& Virmani, R. (2002) Differential accumulation of proteoglycans and hyaluronan in culprit lesions: insights into plaque erosion. Arterioscler. Thromb. Vasc. Biol., 22, $1642-1648$.

Kurtul, A., Yarlioglues, M., Celik, I.E., Duran, M., Elcik, D., Kilic, A., Oksuz, F. \& Murat, S.N. (2015) Association of lymphocyte-to-monocyte ratio with the no-reflow phenomenon in patients who underwent a primary percutaneous coronary intervention for ST-elevation myocardial infarction. Coron. Artery Dis., 26, 706-712.

Muller, J.E., Stone, P.H., Turi, Z.G., Rutherford, J.D., Czeisler, C.A., Parker, C., Poole, W.K., Passamani, E., Roberts, R., Robertson, T., Sobel, B.E., Willerson, J.T. \& Braunwald, E.; the MILIS Study Group (1985) Circadian variation in the frequency of onset of acute myocardial infarction. N. Engl. J. Med., 313, 1315-1322.

Nagueh, S.F., Appleton, C.P., Gillebert, T.C., Marino, P.N., Oh, J.K., Smiseth, O.A., Waggoner, A.D., Flachskampf, F.A., Pellikka, P.A. \& Evangelisa, A. (2009) Recommendations for the evaluation of left ventricular diastolic function by echocardiography. Eur. J. Echocardiogr., 10, 165-193.

Nettelbladt, O., Tengblad, A. \& Hallgren, R. (1989) Lung accumulation of hyaluronan parallels pulmonary edema in experimental alveolitis. Am. J. Physiol., 257, L379-384.

Nunez, J., Nunez, E., Bodi, V., Sanchis, J., Minana, G., Mainar, L., Santas, E., Merlos, P., Rumiz, E., Darmofal, H., Heatta, A.M. \& Llacer, A. (2008) Usefulness of the neutrophil to lymphocyte ratio in predicting long-term mortality in ST segment elevation myocardial infarction. Am. J. Cardiol., 101, 747-752.

Pedicino, D., Vinci, R., Giglio, A.F., Pisano, E., Porto, I., Vergallo, R., Russo, G., Ruggio, A., D’Aiello, A., Flego, D., Annibali, G., Trotta, F., Piacentini, R., Niccoli, G., Liuzzo, G., et al. (2018) Alterations of hyaluronan metabolism in acute coronary syndrome: implications for plaque erosion. J. Am. Coll. Cardiol., 72, 1490-1503.

Ross, R. (1999) Atherosclerosis: an inflammatory disease. $N$. Engl. J. Med., 340, 115-126.

Rovetto, M.J. (1977) Effect of hyaluronidase and methylprednisolone on myocardial function, glucose metabolism, and coronary flow in the isolated ischemic rat heart. Circ. Res., 41, 373-379.

Spinale, F.G. (2007) Myocardial matrix remodeling and the matrix metalloproteinases: influence on cardiac form and function. Physiol. Rev., 87, 1285-1342.

Thygesen, K., Alpert, J.S., Jaffe, A.S., Simoons, M.L., Chaitman, B.R. \& White, H.D.; Joint ESC/ACCF/AHA/WHF Task Force for the Universal Definition of Myocardial Infirction (2012) Third universal definition of myocardial infarction. Circulation, 126, 2020-2035. 
Toole, B.P., Wight, T.N. \& Tammi, M.I. (2002) Hyaluronan-cell interactions in cancer and vascular disease. J. Biol. Chem., 277, 4593-4596.

Triggs-Raine, B. \& Natowicz, M.R. (2015) Biology of hyaluronan: insights from genetic disorders of hyaluronan metabolism. World J. Biol. Chem., 6, 110-120.

Wada, H., Dohi, T., Miyauchi, K., Shitara, J., Endo, H., Doi, S., Konishi, H., Naito, R., Tsuboi, S., Ogita, M., Kasai, T., Hassan, A., Okazaki, S., Isoda, K., Suwa, S., et al. (2017) Preprocedural neutrophil-to-lymphocyte ratio and long-term cardiac outcomes after percutaneous coronary intervention for stable coronary artery disease. Atherosclerosis, 265, 35-40.

Waldenstrom, A., Martinussen, H.J., Gerdin, B. \& Hallgren, R. (1991) Accumulation of hyaluronan and tissue edema in experimental myocardial infarction. J. Clin. Invest., 88, 16221628.

Zimetbaum, P.J. \& Josephson, M.E. (2003) Use of the electrocardiogram in acute myocardial infarction. N. Engl. J. Med., 348, 933-940.

Zimmermann, W.H. (2015) New biologics for the modulation of post-infarct remodeling: matricryptins. J. Am. Coll. Cardiol., 66, 1375-1377. 